\title{
Autopercepção de vulnerabilidade às doenças sexualmente transmissíveis e Aids em mulheres
}

\section{Self-assessment of STD/AIDS vulnerability among women, Brazil}

\author{
Mariângela F Silveira, Jorge U Béria, Bernardo L Horta e Elaine Tomasi \\ Departamento Materno-Infantil e Programa de Pós-Graduação em Epidemiologia, Universidade \\ Federal de Pelotas. Pelotas, RS, Brasil
}

\section{Descritores}

Doenças sexualmente transmissíveis, epidemiologia. Síndrome de imunodeficiência adquirida, epidemiologia. Conhecimentos, atitudes e prática. Percepção.

Mulheres. Correr o risco. Comportamento sexual. Fatores de risco. Saúde da mulher.

\section{Keywords}

Acquired immunodeficiency syndrome, epidemiology. Knowledge, attitudes, practice. Perception. Women. Risktaking. Sex behavior. Risk factors. Sexually transmitted diseases. Women's health.

\section{Resumo}

\section{Objetivo}

Investigar comportamentos de risco e autopercepção de vulnerabilidade às doenças sexualmente transmissíveis (DST) e à Síndrome de imunodeficiência adquirida (Aids) em mulheres.

\section{Métodos}

Dos 281 setores censitários existentes na cidade de Pelotas, RS, foram selecionados 48 a partir de amostragem sistemática. Foi entrevistada uma amostra de 1.543 mulheres, de 15 a 49 anos, por meio de questionário composto de três partes (informações socioeconômicas, perguntas aplicadas em entrevista, questionário auto-aplicado). Para tabulação dos dados, foi utilizado o programa Epi-Info, versão 6.0. Para análise estatística dos dados foram usados o teste de Kappa e a razão de odds.

\section{Resultados}

$\mathrm{Na}$ amostra, 64\% das mulheres achavam impossível ou quase impossível adquirir DST/Aids. Os principais comportamentos de risco foram o não uso de preservativo na última relação antes do depoimento (72\%); início das relações sexuais com menos de 18 anos (47\%); uso de álcool ou drogas pelo parceiro (14\%) ou pela mulher (7\%) antes da última relação; dois ou mais parceiros nos três meses que antecederam o depoimento (7\%) e sexo anal na última relação (3\%); 44\% das mulheres apresentaram dois ou mais comportamentos de risco. A sensibilidade da autopercepção, usando como padrão o escore de risco igual ou superior a dois, foi de $41 \%$. Sua especificidade de $67 \%$.

Conclusões

A autopercepção de vulnerabilidade não é um bom indicador, pois as mulheres não identificam corretamente seu nível de risco.

Abstract

\section{Objective}

To investigate risk behaviors and self-assessment of the vulnerability to sexually transmitted diseases (STD) and acquired immunodeficiency syndrome (AIDS) among women.

\section{Methods}

A systematic sample including 48 of 281 census tracts in the urban area of Pelotas, 
Brazil, was selected. There were interviewed 1,543 women, aged between 15 and 49 years, who had ever been sexually active. Risk behaviors were assessed using a confidential questionnaire that consisted of three sections: socioeconomic profile, interviewers' and self-administered questions. Data analysis was conducted using Epi-Info software, version 6.0. Statistical analysis was carried out using Kappa test and odds ratio.

\section{Results}

Of all women in the sample, 64\% considered impossible or almost impossible for them to acquire an STD or AIDS. The main risk behaviors were non-use of condoms in their last intercourse (72\%); onset of sexual activity before age 18 years (47\%); drug or alcohol use by the partner (14\%) or the woman (7\%) before their last intercourse; two or more partners in the last three months (7\%); and anal sex in the last intercourse (3\%); $44 \%$ reported two or more risk behaviors. Using a risk score of two or more points as the gold standard, sensitivity and specificity of self-assessment were $41 \%$ and $67 \%$, respectively.

Conclusions

Self-assessment of vulnerability is not an adequate indicator of STD and AIDS vulnerability since women do not have an appropriate perception of their risks.

\section{INTRODUÇÃO}

As doenças sexualmente transmissíveis (DST) são freqüentes em todo o mundo. As mulheres representam uma parcela cada vez maior dos casos de HIV/ Aids, ${ }^{13}$ sendo que as DST facilitam a transmissão desse vírus. ${ }^{1}$ Segundo a Unaids ${ }^{20}$ em dezembro de 1999 havia 34,3 milhões de pessoas vivendo com o HIV em todo o mundo. Dos infectados, 15,7 milhões eram mulheres, o que correspondia a quase metade de todas as pessoas contaminadas em1999. Grande parte das mulheres se contaminava na faixa etária entre 15 e 24 anos, em plena vida reprodutiva, o que eleva o número de casos entre crianças devido à transmissão vertical.

Na América Latina, cerca de 1,3 milhões de pessoas estão contaminadas. Entre os adultos, $25 \%$ são mulheres.* De acordo com dados do Ministério da Saúde, presume-se que no Brasil o número de portadores ultrapasse 500 mil. De 1980 até maio de 2000, mais de 190.000 casos foram registrados. Dos casos acumulados, $25 \%$ ocorreram em mulheres - cerca de metade delas na faixa etária dos 20 a 34 anos. Atualmente, entre os casos novos de Aids, há dois homens para cada mulher. A participação feminina na doença tem, portanto, aumentado.**

As mulheres são especialmente vulneráveis às DST por características biológicas: a superfície vaginal exposta ao sêmen é relativamente extensa, e o sêmen apresenta maior concentração de HIV do que o líquido vaginal. As DST são mais freqüentemente assintomáticas; e a mucosa vaginal é frágil, princi- palmente em mulheres mais jovens. ${ }^{1} \mathrm{O}$ papel social, ou de gênero, da mulher, também aumenta seu risco. As relações desiguais de poder e a dependência econômica das mulheres, especialmente em países em desenvolvimento, limitam o acesso a informações adequadas e atualizadas. Quando isto ocorre é penosa a modificação de comportamentos e a manutenção dessas mudanças nas interações cotidianas. ${ }^{1} \mathrm{Pe}-$ los motivos mencionados e em razão da proporção crescente de mulheres entre os casos novos de Aids, é importante que sejam desenvolvidas estratégias de prevenção mais efetivas entre as mulheres. No campo da educação, o modelo das crenças em saúde (health belief models) preconiza que há quatro percepções relevantes para aceitar intervenções médico-sanitárias: de suscetibilidade, severidade, benefícios e barreiras. Indivíduos que não se sentem vulneráveis a uma doença não costumam aceitar as medidas preventivas recomendadas. ${ }^{8}$ Entre estudos que aplicaram o modelo das crenças em saúde para a questão de DST/Aids, alguns achados mostraram maior percepção de susceptibilidade associada à redução de comportamentos de risco. ${ }^{9}$ Outros observaram que, apesar de conscientes de sua vulnerabilidade, muitos indivíduos persistiam em práticas de risco e, conseqüentemente, em se contaminar. ${ }^{12}$

Beria et $\mathrm{al}^{2}$ realizou estudo sobre a autopercepção de vulnerabilidade para DST/HIV em adolescentes escolares. Outro estudo empregou o modelo de crenças em saúde para investigar a percepção de risco de pessoal de enfermagem em relação à contaminação por seringas usadas. ${ }^{4}$ Não foi identificado nenhum estudo populacional sobre a autopercepção de 
vulnerabilidade em adultos, no Brasil. Há, portanto, necessidade de se conhecer a percepção de vulnerabilidade para adquirir uma DST, com a finalidade de instrumentalizar formas de intervenções preventivas.

O presente artigo teve como objetivo avaliar a prevalência de comportamentos de risco para DST/Aids em mulheres e relacioná-la com a autopercepção de vulnerabilidade. As hipóteses do estudo foram: 1) há mulheres que não percebem adequadamente sua situação de vulnerabilidade para DST/HIV; 2) a percepção de vulnerabilidade estaria associada a uma menor ocorrência de comportamentos de risco para DST/HIV e; 3) a percepção correta de vulnerabilidade seria maior em mulheres de melhor situação socioeconômica e maior escolaridade.

\section{MÉTODOS}

Pelotas é um município localizado no extremo Sul do Brasil, com população, em 2001, constituída de 323.034 habitantes, dos quais 169.854 eram mulheres. O Serviço de Assistência Especializada em Aids desse município, que assiste a grande maioria dos casos diagnosticados de Aids, contava no início de 2001 com 425 homens e 256 mulheres registradas, numa razão de 1,66.

O presente estudo* teve delineamento transversal. As mulheres de 15 a 49 anos residentes na área urbana de Pelotas foram a população alvo. Com uma amostra em torno de 1.500 mulheres seria possível estimar qualquer prevalência com um erro aceitável de três pontos percentuais. Para localizar 1.500 mulheres, seria necessário visitar 1667 domicílios. Assim, para definição da população estudada, foram selecionados 48 setores censitários, dos 281 existentes em Pelotas, a partir de amostragem sistemática.

Este procedimento garantiu uma amostra que representasse todos os setores da cidade. Em cada setor foi sorteado quarteirão, esquina e domicílio de início. As entrevistadoras percorreram a vizinhança no sentido horário, selecionando um domicílio a cada três até completar 44 domicílios. Quarteirões adicionais já estavam previamente sorteados para completar a cota de domicílios, se necessário.

Foram selecionados 2.112 domicílios, e identificadas 1.851 mulheres, das quais $65(3,5 \%)$ recusaram ou não puderam ser entrevistadas após no mínimo três tentativas. No caso de domicílios fechados, a informação sobre idade e sexo dos moradores foi obtida por meio de vizinhos. Das mulheres entrevista- das, 1.543 relataram atividade sexual e passaram a constituir a amostra do estudo. Dessas, 110 (7\%) foram visitadas mais uma vez, sendo repetida a questão sobre a chance de adquirir DST/Aids, obtendo-se um Kappa ponderado de 0,74.

Foram selecionadas e treinadas 30 entrevistadoras com segundo grau completo. $\mathrm{O}$ treinamento incluiu técnicas de entrevista e de localização dos domicílios, leitura dos questionários e dos manuais de instruções, dramatizações, prova teórica (após o treinamento) e avaliação de desempenho no campo. Realizou-se um estudo piloto no final do treinamento. $\mathrm{O}$ trabalho de campo foi realizado de outubro de 1999 a janeiro de 2000 .

A coleta de dados foi feita por meio de questionário composto de três partes: um módulo domiciliar com informações socioeconômicas, um módulo individual, com perguntas aplicadas pela entrevistadora, e um questionário confidencial auto-aplicado, no qual não constava o nome da entrevistada, mas apenas um número de identificação. O questionário auto-aplicado, depois de preenchido, era colocado pela entrevistada dentro de um envelope que ela mesma lacrava. O envelope era posteriormente aberto e codificado pela supervisora geral do estudo. Foi obtido consentimento prévio de todas as entrevistadas para participação na pesquisa.

As seguintes variáveis foram coletadas: idade (em anos completos); cor (observada pela entrevistadora e classificada como branca ou parda/negra); estado civil (casada/com companheiro, solteira, viúva ou separada/divorciada); renda familiar e da mulher (em reais, referente ao último mês, posteriormente transformada em salários mínimos); renda per capita (obtida dividindo-se a renda familiar pelo número de moradores do domicílio); escolaridade (em anos completos com aprovação); prática de religião (sim ou não); níveis de autopercepção de vulnerabilidade para DST/Aids (muito possível, possível, pouco possível, quase impossível ou impossível).

As variáveis utilizadas para medir os comportamentos de risco foram coletadas no questionário auto-aplicado: 1) idade de início das relações sexuais (definidas como relação anal, vaginal ou oral, definiu-se como sendo de risco quando iniciadas antes dos 18 anos, conforme a maioria dos estudos constantes na literatura) $;{ }^{15}$ 2) uso de preservativos na última relação sexual antes da entrevista (sim ou não). 3) uso de álcool pela mulher antes da última relação (sim ou não) ou pelo parceiro (sim ou não); 4) uso de drogas antes da última 
relação pela mulher (sim ou não) ou pelo parceiro (sim ou não); 5) sexo anal na última relação (sim ou não); e 6) número de parceiros nos últimos três meses (definiu-se como de risco o relato de dois ou mais parceiros). Um escore de risco foi construído pela soma das seis variáveis acima, codificadas como zero (ausente) ou um (presente). A escolha das variáveis que avaliaram comportamentos de risco seguiu dois critérios: ampla utilização em outros estudos, conforme a revisão da literatura e adequação a questionário auto-aplicado para a população em geral (incluindo mulheres com baixa escolaridade).

O controle de qualidade do trabalho de campo foi feito por 12 supervisores, cada um responsável por quatro setores. Os supervisores repetiam no mínimo $5 \%$ das entrevistas e revisavam todos os questionários preenchidos. Todos os questionários foram digitados duas vezes no programa Epi-Info 6.0 por profissionais diferentes, o que permitiu a comparação dos bancos de dados e a correção de erros de digitação. A checagem dos dados incluiu verificações de consistência e amplitude das variáveis. Para a análise dos dados, foi utilizado o programa SPSS 8.0, realizando-se distribuição de freqüências, teste do quiquadrado para associações, análise de variância para variáveis contínuas e regressão logística não condicional para detectar interações. Foram consideradas como significativas as interações com teste de significância menor ou igual a $10 \%$. Os valores ignorados para as variáveis independentes foram recodificados para a categoria modal das variáveis agrupadas. A variável com maior percentual de valores ignorados foi a renda familiar, $\operatorname{com} 2,1 \%$.

Devido à presença de valores ignorados nas variáveis que integravam o escore de risco, cerca de $11 \%$ das mulheres não puderam ser classificadas na análise inicial. Para reduzir este percentual, as seguintes medidas foram adotadas: as idades de início da atividade sexual ignoradas $(5,2 \%$ das mulheres) foram estimadas por regressão linear utilizando-se as variáveis idade, escolaridade e renda familiar. Quanto ao número de parceiros nos três meses anteriores à entrevista, não houve informação para $4,9 \%$ das mulheres. Como não foi possível obter uma equação de regressão com um bom ajuste para essa variável, multiplicou-se a soma dos outras cinco componentes do escore de risco por 1,2 (efeito médio do número de parceiros). Restaram 3,4\% de valores ignorados para uma ou mais variáveis integrantes do escore final. Esses casos foram excluídos da análise.

\section{RESULTADOS}

A Tabela 1 mostra as distribuições das variáveis socioeconômicas e demográficas. Verifica-se que a distribuição dos grupos foi homogênea em relação à idade, com uma menor freqüência de adolescentes $(9,7 \%) ; 79 \%$ das mulheres eram de cor branca; $61 \%$ estavam casadas ou em união; $19 \%$ tinham renda familiar per capita igual ou inferior a cinco salários mínimos; $41 \%$ das mulheres declararam não ter nenhuma renda própria; pouco mais de metade tinham até oito anos de escolaridade, e 55\% não praticavam religião.

Quanto à autopercepção de vulnerabilidade para DST/Aids, $40 \%$ das mulheres consideraram "impossível" adquirir a doença, $24 \%$ consideraram "quase impossível", enquanto $18 \%$ classificaram a contaminação como"pouco possível". As categorias "muito possível" e "possível" foram agrupadas, pois havia apenas 54 mulheres (4\%) na primeira e 219 (14\%) na segunda. Percebe-se que a maior parte das mulheres (64\%) considerava impossível ou quase impossível adquirir uma DST ou Aids.

A Tabela 2 mostra a freqüência dos comportamentos de risco para DST/Aids. A idade de início das relações sexuais foi inferior a 18 anos em $47 \%$ da amostra; $72 \%$ não usaram preservativo na última re-

Tabela 1 - Caracterização da amostra de mulheres que já mantiveram relações sexuais segundo fatores demográficos e socioeconômicos. Pelotas, 1999-2000.

\begin{tabular}{|c|c|c|}
\hline Variável & Freqüência* & $\%$ \\
\hline \multicolumn{3}{|c|}{ Idade da mulher em anos completos } \\
\hline $15-19$ & 150 & 9,7 \\
\hline $20-24$ & 240 & 15,6 \\
\hline $25-29$ & 214 & 13,9 \\
\hline $30-34$ & 212 & 13,7 \\
\hline $35-39$ & 238 & 15,4 \\
\hline $40-44$ & 240 & 15,6 \\
\hline $45-49$ & 249 & 16,1 \\
\hline \multicolumn{3}{|l|}{ Cor da pele } \\
\hline Branca & 1.216 & 78,8 \\
\hline Parda/preta & 327 & 21,2 \\
\hline \multicolumn{3}{|l|}{ Estado civil } \\
\hline Casada/com companheiro & 937 & 60,8 \\
\hline $\begin{array}{l}\text { Solteira } \\
\text { Som }\end{array}$ & 422 & 27,3 \\
\hline Viúva & 34 & 2,2 \\
\hline Separada/divorciada & 150 & 9,7 \\
\hline \multicolumn{3}{|c|}{ Renda per capita em salários-mínimos } \\
\hline $0,0-0,5$ & 280 & 18,7 \\
\hline $0,51-1,00$ & 348 & 23,0 \\
\hline $1,01-2,00$ & 395 & 26,2 \\
\hline Maior que 2 & 487 & 32,1 \\
\hline \multicolumn{3}{|c|}{ Renda da mulher em salários-mínimos } \\
\hline Nenhuma & 631 & 41,2 \\
\hline Igual ou menor que 1 & 257 & 16,7 \\
\hline $1,01-3,0$ & 364 & 23,7 \\
\hline Maior que 3,0 & 283 & 18,4 \\
\hline \multicolumn{3}{|c|}{ Escolaridade da mulher em anos completos } \\
\hline $0-4$ & 279 & 18,2 \\
\hline $5-8$ & 573 & 37,2 \\
\hline $9-11$ & 406 & 26,4 \\
\hline 12 ou mais & 279 & 18,2 \\
\hline \multicolumn{3}{|l|}{ Prática de religião } \\
\hline Não & 849 & 55,0 \\
\hline Sim & 694 & 45,0 \\
\hline
\end{tabular}
mas mulheres. 
lação sexual e sete por cento das mulheres usaram álcool e/ou drogas antes da última relação sexual anterior à entrevista. Três por cento da amostra praticou sexo anal na última relação; sete por cento teve dois ou mais parceiros nos últimos três meses; e 13,5\% dos parceiros usaram álcool e/ou drogas antes da última relação sexual.

A Figura mostra a distribuição do escore de risco, sendo que $10 \%$ das mulheres tinham escore igual a zero - não apresentaram nenhum comportamento de risco - e $44 \%$ apresentaram escore igual ou maior do que dois. Apenas sete mulheres $(0,4 \%)$ apresentaram escore maior ou igual a cinco.

A Tabela 3 mostra que, nos grupos de mulheres que consideraram possível adquirir DST/Aids, o escore médio de risco foi maior do que nas mulheres que o consideraram impossível. Esta diferença, apesar de pequena, foi estatisticamente significante. Para o escore dicotomizado, com um ponto de corte igual a dois comportamentos, a associação foi mantida. A razão de odds de apresentar um escore de risco maior ou igual a dois foi cerca de $50 \%$ maior nos grupos que consideravam possível adquirir DST/Aids.

A Tabela 4 mostra que a sensibilidade da autopercepção, usando como padrão o escore de ris- co, foi de $41 \%$, e sua especificidade de $67 \%$. Essa análise foi repetida com outros pontos de corte para $\mathrm{o}$ escore de risco (padrão): para o ponto de corte igual a um, a sensibilidade foi de $37 \%$ e a especificidade de $69 \%$, enquanto que para o ponto de corte igual a três desses valores, a sensibilidade foi de $49 \%$ e a especificidade de $65 \%$.

$\mathrm{Na}$ Tabela 5 tentou-se identificar possíveis modificadores de efeito que pudessem melhorar a sensibilidade da autopercepção. Conforme o teste de

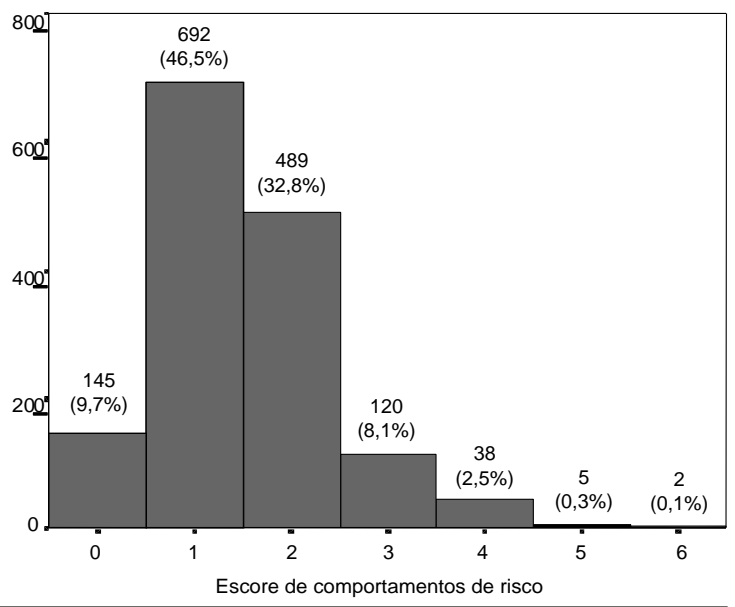

$\overline{\text { Figura - Número de mulheres conforme o escore de risco }}$ para DST/AIDS. Pelotas, 1999-2000.

Tabela 2 - Freqüência dos comportamentos de risco para DST/Aids em mulheres. Pelotas, 1999-2000.

\begin{tabular}{lrr}
\hline Variável & Freqüência & $\%$ \\
\hline Idade de início das relações sexuais em anos & & 774 \\
ou $=18$ & 688 & 52,9 \\
$<$ ou $=17$ & 1.097 & 71,9 \\
Uso do preservativo na última relação sexual & 428 & 28,1 \\
Não & 1.426 & 93,1 \\
Sim & 106 & 6,9 \\
Uso de álcool e/ou droga pela mulher antes da última relação sexual & 1.480 & 97,2 \\
Sim & 42 & 2,8 \\
Prática de sexo anal na última relação sexual & 1.367 & 93,1 \\
Simo & 101 & 6,9 \\
Número de parceiros nos últimos três meses & 1.305 & 86,5 \\
Um ou menos & 203 & 13,5 \\
Dois ou mais & & \\
Uso de álool ou droga pelo parceiro antes da última relação sexual & \\
Sim & &
\end{tabular}

DST/Aids: Doenças sexualmente transmissíveis e Aids.

Tabela 3 - Escores de comportamentos de risco conforme a autopercepção de vulnerabilidade em mulheres. Pelotas, 19992000

\begin{tabular}{|c|c|c|c|}
\hline $\begin{array}{l}\text { Autopercepção de } \\
\text { vulnerabilidade para } \\
\text { DST/AIDS }\end{array}$ & $\begin{array}{l}\text { Escore médio de risco } \\
\qquad \text { (desvio-padrão)* }^{*}\end{array}$ & $\begin{array}{l}\text { Escore de risco } \\
\qquad \geq 2^{* *}\end{array}$ & $\begin{array}{c}\text { Razão de odds para } \\
\text { escore de risco } \geq 2 \\
\text { (IC } 95 \%)\end{array}$ \\
\hline $\begin{array}{l}\text { Muito possível/possível } \\
\text { Pouco possível } \\
\text { Quase impossível } \\
\text { Impossível }\end{array}$ & $\begin{array}{l}1,63(0,99) \\
1,62(0,96) \\
1,41(0,85) \\
1,43(0,86)\end{array}$ & $\begin{array}{l}49,1 \% \\
50,0 \% \\
42,3 \% \\
40,1 \%\end{array}$ & $\begin{array}{r}1,44(1,07-1,93) \\
1,49(1,11-2,00) \\
1,09(0,84-1,42) \\
1,00\end{array}$ \\
\hline
\end{tabular}

*Análise de variância: $p=0,002$; teste para linearidade $p=0,001$.

**Teste do Qui-quadrado para heterogeneidade: $p=0,025$; teste do Qui-quadrado para tendência linear: $p=0,004$. 
interação, as únicas variáveis que possivelmente aumentariam a sensibilidade foram a maior escolaridade e a prática de religião, ambas com valor p igual a 0,08 .

\section{DISCUSSÃO}

O presente estudo mostra ser possível investigar comportamentos de risco para DST/Aids em mulheres por meio de um delineamento transversal no Brasil. A amostra representativa da população e o baixo percentual de perdas reduzem a possibilidade de viés de seleção.

Como possíveis limitações desse estudo estão 1) a dificuldade em relatar experiências íntimas mesmo com questionário auto-aplicado; 2) a realização das entrevistas no domicílio, o que pode ter inibido as respondentes, especialmente as adolescentes; 3) o fato de avaliar apenas comportamentos referentes à última relação anterior à entrevista e 4) a baixa escolaridade de algumas respondentes. Estas limitações teriam um efeito conservador sobre o resultado do estudo, diminuindo a prevalência de risco. Outras possíveis limitações do estudo incluem a avaliação da autopercepção por meio de uma única pergunta e a atribuição de pesos iguais para os seis comportamentos incluídos no escore de risco. Esta opção foi devida à inexistência de critérios objetivos para definir diferentes pesos para cada comportamento. Sugerese a realização de estudos posteriores com maior detalhamento tanto do escore de risco quanto da avaliação de autopercepção.

Uma outra limitação possível teria sido a não estratificação dos resultados conforme o tipo de parceiro da última relação sexual (fixo ou ocasional). Um número crescente de mulheres brasileiras está sendo contaminado por seus parceiros fixos. Das mulheres diagnosticadas com Aids em 2001, 56\% não tinham história de múltiplos parceiros. ${ }^{11}$ A questão abordada (como a mulher se protegeu na ultima relação) pareceu mais relevante do que a existência ou não de relacionamento afetivo. A literatura mostra que intervenções tendem a ser menos efetivas com parceiros fixos do que com ocasionais. ${ }^{17}$

Tabela 4 - Sensibilidade e especificidade da autopercepção de vulnerabilidade para DST/AIDS em relação ao escore de comportamentos de risco. Pelotas, 1999-2000.

Autopercepção de $\quad$ Escore $0-1,99 \quad$ Escore $\geq 2$ vulnerabilidade para

DST/Aids

\begin{tabular}{lcc}
\hline Possível & $32,7 \%$ & $40,7 \%$ \\
Impossível & $67,3 \%$ & $59,3 \%$ \\
\hline Total & 826 & 651 \\
\hline
\end{tabular}

Em relação à autopercepção de vulnerabilidade, $18 \%$ das entrevistadas pensavam ser possível ou muito possível adquirir DST/Aids.Embora baixo, o percentual é maior do que o observado em outros estudos. Em um estudo na República Dominicana, $11 \%$ de mulheres marginalizadas se declararam em risco de adquirir uma DST. ${ }^{5}$ Em Pelotas (1995), entre meninas escolares de 14 a 19 anos, oito por cento acreditavam ser possível adquirir Aids e 1\% muito possível. ${ }^{2}$ No presente estudo, no grupo entre 15 e 19 anos, $13 \%$ pensavam ser possível e $6 \%$ muito possível, o que pode significar que campanhas realizadas nas escolas e mídia tenham sensibilizado as adolescentes sobre o risco da Aids, embora os dados se refiram a grupos ligeiramente diferentes.

Quanto ao início precoce da atividade sexual, observou-se no presente estudo que $47 \%$ das mulheres já mantinham relações sexuais antes dos 18 anos. Na América Latina (1998), o percentual foi de $56 \%$ aos 20 anos. No Brasil (1998), 14\% a 34\% das mulheres já estavam casadas ou em união antes dos 18 anos, conforme a região: ${ }^{18}$ em Pelotas (1995), 45\% das mulheres entre 15 e 19 anos já tinham iniciado atividade sexual. ${ }^{13}$ Embora existam diferenças quanto à definição dos indicadores, todos os estudos indicam altas prevalências de início precoce da vida sexual. As diferenças entre os estudos são certamente devidas a aspectos culturais.

Em relação à prática de sexo anal, foi encontrada uma prevalência de $3 \%$ na última relação sexual antes da realização da entrevista. Na literatura, essas prevalências variam, 7\% quando investigado o último ano, ${ }^{*} 7 \%$ quando da última relação (adolescentes escolares). ${ }^{3}$ Outros estudos utilizaram métodos

Tabela 5 - Associação entre a autopercepção de vulnerabilidade para DST/AIDS e o escore de comportamentos de risco, estratificado conforme características das mulheres. Pelotas, 1999-2000.

\begin{tabular}{lcc}
\hline $\begin{array}{l}\text { Variável modificadora } \\
\text { de efeito }\end{array}$ & Sensibilidade & Especificidade \\
\hline Cor & & \\
$\quad$ Branca & $\mathrm{p}=0,84$ & \\
$\quad$ Parda/negra & $40,4 \%$ & $67,8 \%$ \\
Idade & $41,7 \%$ & $65,4 \%$ \\
$\quad<35$ & $\mathrm{p}=0,81$ & \\
$\geq 35$ & $41,7 \%$ & $65,2 \%$ \\
Escolaridade & $38,9 \%$ & $69,0 \%$ \\
$\quad \leq 8$ & $\mathrm{p}=0,08 \%$ & \\
$>8$ & $37,3 \%$ & $66,9 \%$ \\
Renda da mulher & $45,5 \%$ & $67,9 \%$ \\
$\leq 3$ SM & $\mathrm{p}=0,13$ & \\
$\quad>3$ SM & $38,7 \%$ & $68,0 \%$ \\
Prática de religião & $52,7 \%$ & $65,0 \%$ \\
$\quad$ Não & $\mathrm{p}=0,076$ & \\
Sim & $38,4 \%$ & $65,7 \%$ \\
Estado civil & $44,0 \%$ & $69,2 \%$ \\
$\quad$ Casada/com companheiro & $\mathrm{p}=0,71$ & \\
$\quad 37,1 \%$ & $69,7 \%$ \\
Não casada & $46,0 \%$ & $68,3 \%$ \\
\hline
\end{tabular}

*Fonte: http://www.unAids.org/epidemic_update/report/epi_core.ppt [Disponível em URL: 2001 fev 26 ] 
distintos (questionário auto-aplicado, entrevista por telefone) sendo que a natureza da pergunta, que entra na intimidade do entrevistado, pode levar a muitas respostas falso-negativas.

Encontrou-se uma prevalência de $28 \%$ de uso de preservativos masculinos na última relação sexual. Em Pelotas, este índice foi de $42 \%$ para adolescentes escolares. ${ }^{3} \mathrm{O}$ uso de preservativos, apesar de várias campanhas, ainda é baixo, variando de 3,2\% a 22,5\% em alguns estudos isolados. ${ }^{5,7,10,14}$ No Brasil, em 1996, encontrou-se uma prevalência de uso na última relação de risco de $30,8 \%$ em homens e de $11,4 \%$ em mulheres, na faixa etária de 15 a 49 anos. ${ }^{19}$ Um estudo brasileiro de base populacional, com uma população entre 16 e 65 anos, mostrou uma prevalência de uso de preservativo nos últimos 12 meses que precederam a pesquisa de $24 \%$ (64\% em relações eventuais). Na faixa etária de 16 a 25 anos, o índice foi de $44 \%$ ( $53 \%$ entre os homens e $35 \%$ entre as mulheres). No geral, o grupo que menos utilizou o preservativo foram as mulheres com parceria estável. ${ }^{6}$ As diferenças entre os estudos são provavelmente devidas a diferenças metodológicas, à disponibilidade de preservativos e à intensidade das campanhas preventivas. A baixa prevalência encontrada no presente estudo é extremamente preocupante, principalmente quando se sabe que o uso do preservativo é o único método realmente efetivo na prevenção das DST.

Encontrou-se que 7\% das mulheres mantiveram relações com dois ou mais parceiros nos últimos 3 meses. Nos EUA, entre mulheres solteiras de 15 a 44 anos, este índice variou de $6,6 \%{ }^{16} 8,4 \% .^{15}$

Quanto à avaliação se a autopercepção de vulnerabilidade reduz os comportamentos de risco, observou-se que tanto o escore de risco médio como a razão de odds foram maiores nos grupos de mulheres que consideraram possível adquirir DST/Aids. Isto sugere que o fato de se perceber em risco não determina a mudança de comportamento. Sobre se o nível de risco influenciaria a autopercepção, observou-se que a sensibilidade da autopercepção é baixa, pois apenas $41 \%$ (IC 95\%: 37-45\%) das mulheres classificadas como de risco assim se identificaram. A especificidade foi de 67\% (IC 95\%: 63-71\%). Identificou-se também um grupo importante de mulheres com percepção inadequada $-59 \%$ das mulheres classificadas como de maior risco consideraram impossível adquirir uma DST ou Aids. Os resultados das Tabelas 3 e 4 poderiam parecer incompatíveis, mas não são. Na Tabela 3, observa-se um aumento discreto no escore de risco quando a mulher se percebe em risco "possível ou muito possível" de adquirir DST/Aids, mas a diferença entre os grupos extremos é de apenas 0.2 comportamento de risco. A Tabela 4 confirma que esta associação é significativa, mas de fraca intensidade, uma vez que a sensibilidade e especificidade são baixas.

Os dados apresentados são importantes já que mostram que a prevalência de comportamentos de risco é alta na população de mulheres em geral, e que a autopercepção de risco é muito baixa e freqüentemente inadequada. A partir desses resultados, pode-se planejar intervenções preventivas mais adequadas à realidade e, portanto, mais efetivas.

Em resumo, o estudo mostrou que a maior parte das mulheres apresenta um ou dois comportamentos de risco. Os comportamentos mais comuns são o início precoce da atividade sexual, o não uso de preservativos e uso de álcool/drogas pelo parceiro. O estudo também mostrou que a autopercepção de vulnerabilidade não é um bom indicador, pois as mulheres não identificam corretamente seu nível de risco.

A prevenção das doenças sexualmente transmissíveis e da Aids entre as mulheres depende de sua conscientização da existência do risco. A autopercepção adequada contribuiria para ajudá-las a evitar comportamentos e situações de risco, assim como adotar rotineiramente medidas preventivas, como o uso de preservativos nas relações sexuais.

\section{AGRADECIMENTOS}

Aos colegas do consórcio de pesquisa, ao Professor Cesar Victora da Faculdade de Medicina da Universidade Federal de Pelotas, por suas sugestões e comentários.

\section{REFERÊNCIAS}

1. Bastos Fl, Szwarcwald CL. AIDS and pauperization: principal concepts and empiricalevidence. Cad Saúde Pública 2000;16:65-76.

2. Béria J, Oliveira OMF, Carret MLV. Adolescentes e DST/AIDS: pesquisa formativa em um estudo de intervenção. In: Béria J. Ficar, transar ...a sexualidade do adolescente em tempos de AIDS. Porto Alegre: Tomo Editorial; 1998. p. 37-54. 
3. Béria J, Barros AJD. Oito meses depois: o impacto da intervenção entre os adolescentes. In: Béria J. Ficar, transar ... a sexualidade do adolescente em tempos de AIDS. Porto Alegre: Tomo Editorial; 1998. p. 111-25.

4. Brevidelli MM, Cianciarullo TI. Application of the health belief model to the prevention of occupationalneedle accidents. Rev Saúde Pública 2001;35:193-201.

5. Brewer TH, Hasbun J, Ryan CA, Hawes SE, Martinez S, Sanchez J, et al. Migration, ethnicity and environment: HIV risk factors for women on the sugar cane plantations of the Dominican Republic. AIDS 1998;12:1879-87.

6. [CEBRAP/MS] Centro Brasileiro de Análise e Planejamento, Ministério da Saúde. Projeto Comportamento Sexual da População Brasileira e Percepções do HIV/ Aids: relatório de pesquisa [on line]. Disponível em URL: http://www.aids.gov.br/cebrap/ relatorio_pesquisa_cebrap.htm [2001 fev 26].

7. DeHovitz JA, Kelly P, Feldman J, Sierra MF, Clarke L, Bromberg J et al. Sexually transmitted diseases, sexual behavior, and cocaine use in inner-city women. Am J Epidemiol 1994;140:1125-34.

8. Janz, NK, Becker MH. The health belief model: a decade later. Health Educ Q 1984;11:126-32.

9. McBride DC, Weatherby NL, Inciardi JÁ, Gillespie AS. AIDS susceptibility in a migrantpopulation: perception and behavior. Subst Use Misuse 1999;34:633-52.

10. Michael RT, Wadsworth J, Feinleib J, Johnson AM, Laumann EO, Wellings K. Private sexual behavior, public opinion, and public health policy related to sexually transmitted diseases: a US-British comparison. Am J Public Health 1998;88:749-54.

11. Ministério da Saúde. Coordenação Nacional de DST e Aids. Epidemiologia [on line] Disponível em URL: http://www.Aids.gov.br/cgi/deftohtm.exe?Aids.def [2002 jan 18].
12. Momas I, Helal H, Pretet S, Marsal L, Poinsard, R. Demographic and behavioral predictors of knowledge and HIV seropositivity: results of a survey conducted in threeanonymous and free counselling and testing centers. Eur J Epidemiol 1997;13:255-60.

13. Olinto MTA, Galvão LW. Características reprodutivas de mulheres de 15 a 49 anos: estudos comparativos e planejamento de ações. Rev Saúde Pública 1999;33:64-72.

14. Roye CF. Condom use by Hispanic and AfricanAmerican adolescent girls who usehormonal contraception. J Adolesc Health 1998;23:205-11.

15. Seidman SN, Mosher WD, Aral SO. Predictors of high-risk behavior in unmarried Americanwomen: adolescent environment as risk factor. J Adolesc Health 1994;15:126-32.

16. Seidman SN, Mosher WD, Aral SO. Women with multiple sexual partners: United States, 1988. Am J Public Health 1992;82:1388-94.

17. Shepherd J, Weston R, Peersman G, Napuli IZ. Cervical cancer and sexual lifestyle: a systematic reviewof health education interventions targeted at women. Health Educ Res 2000;15:681-94.

18. The Alan Guttmacher Institute. Rumo a um novo mundo: a vida sexual e reprodutiva de mulheres jovens. New York: The Alan Guttmacher Institute; 1998.

19. UNAIDS. Brazil: Epidemiological fact sheet on HIV/ AIDS and sexually transmitted diseare - 2000 update [on line]. Disponível em URL: http://www.unAids.org/ hivAidsinfo/statistics/june00/fact_sheets/pdfs/brazil.pdf [2001 fev 26]. 8Janz, NK, Becker MH. The health belief model: a decade later. Health Educ $Q$ 1984;11:126-32.

20. UNAIDS. Report on the global HIV/AIDS epidemic. Geneva; 2000. 